\title{
International Education Collaboration Using Social Media in Teaching and Learning Pain Management
}

\author{
Mimi Tse ${ }^{1 *}$, Nada Lukkahatai ${ }^{2}$, Kin Cheung ${ }^{1}$, Angel Tang ${ }^{1}$, Shamay $\operatorname{Ng}^{1}$ and Rick Kwan ${ }^{1}$ \\ ${ }^{1}$ School of Nursing, The Hong Kong Polytechnic University, Hong Kong \\ ${ }^{2}$ School of Nursing, Johns Hopkins University, USA
}

*Corresponding author: Mimi Tse, Associate Professor, School of Nursing, The Hong Kong Polytechnic University, Hong Kong

\begin{tabular}{l}
\hline ARTICLE INFO \\
Received: 幽 June 24, 2019 \\
Published: 幽 July 02, 2019 \\
\hline \\
Citation: Mimi Tse, Nada Lukkahatai, Kin \\
Cheung, Angel Tang, Shamay Ng, et al., \\
International Education Collaboration \\
Using Social Media in Teaching and \\
Learning Pain Management. Biomed \\
J Sci \& Tech Res 19(2)-2019. BJSTR. \\
MS.ID.003286.
\end{tabular}

Keywords: Social Media; Traditional Chinese Medicine; Education; Pain

\begin{abstract}
All health-related professionals encounter patients with pain throughout their career. Pain is also a culturally moderated phenomenon. This project describes a journey of using social media to enhance the learning experience in pain \& symptoms management among master students from the two nursing schools. For this collaborative effort, faculties from the two institutes set up a Facebook closed group page as a tool for collaboration. The faculties uploaded videos on the use of non-pharmacological strategies and Chinese Traditional Medicines. Students were invited to join, share and discuss the pain management topics on the Facebook closed group page from October to December 2018. Students were asked to reflect on their learning experience at the end. Seventy-nine nursing students participated in the sharing and discussion. Students from both schools treasured this international learning experience and enjoyed the flexibility of using social media. They actively participated in the discussion and sharing relevant articles and photos and local news related to topics. Students found this activity rewarding and widening the cultural sensitivity perspective. As health becomes a global issue, the distance collaborations among health care professionals are common. Social media platforms can be used as tools to overcome scheduling issues and encourage interaction and deliberation. However, this project is limited by specific social media (Facebook). Not all students are regular Facebook users. For the future collaboration, we will need to explore other social media options.
\end{abstract}

In conclusion, the use of social media embraces the technology-assisted approaches and enhancing an effective international teaching and learning experience to students, the teaching team will continue to develop and refine collaborative mode to facilitate students learning.

\section{Introduction}

Pain management is a fundamental to nursing care for most of patients with chronic conditions. In addition to provide traditional care, nurses are required to master skills in interpreting and applying new knowledge based on best available evidence. Nursing research and evidence-based practice course is offered in most nursing schools to expose nursing students to research and evidence-based practice. Pain, however, is a culturally moderated phenomenon. The international collaboration in teaching and learning in this specific topic can lead to an exposure to new approaches and exchange new perspectives in pain management. It can enhance student's engagement and cultural sensitivity. International knowledge exchanges between faculties and students have been identified as one strategy to strengthen nursing care, education and research [1] A key challenge of most international collaboration is travel cost and time. Social media, including Facebook, Twitter, and Instagram, has been part of our daily media consumption routines worldwide. Recently it has become one of the most effective tools in communications, marketing, News, electronic publishing etc. [2]. In academia, social media becomes one of communication methods tools used among students and faculties in many universities/ institutions. 
It has been found to be useful in making announcements, services of advising, educational activities, marketing, personal postings, expressing opinions and News [3]. From an educational perspective, advances in technology and the internationalization of curriculum are possible with the increasing use of information technology and social media. With these in mind, we are examining the feasibility in using social media in international education collaboration on a specific pain management topic for post graduate nursing students from two universities.

\section{Teaching and Learning}

Pain and Symptoms Management Course (School of Nursing in Hong Kong): Traditional Chinese medicine (TCM) is culturally accepted and widely used in Hong Kong, Taiwan and other Asian countries [4]. The prevalence of TCM gradually increased from 2000 to 2009, and acupuncture, Chinese herbal medicine and moxibustion are commonly used. Indeed, the use of TCM warrant further use among various populations. In this regard, health care professions, who are with the patients $24 / 7$ would be in a good position to learn more about TCM, and, transfer of their knowledge and understanding to their clients in order to manage various health conditions, as well as their pain situations in particular. Students ( $\mathrm{n}=69$; divided into 7 groups) taking the course Pain \& Symptom Management were invited to join Chinese Traditional Medicines (TCM) Facebook activities. Upon completion of the course, ie, in Dec 2018, Facebook discussion measured by number of entries, content of discussion, the use of relevant and update literature for the students. Students submitted their reflection on what went well and what need to be done to improve this collaborative activity [5]

Evidence Based Practice Project on Pain Management (School of Nursing in USA): As a part of graduate nursing program requirement, students are required to complete a research and evidence-based practice in nursing course. The evaluation for student's achievement in this course includes a final group project, which consist of

a) select and develop a clinical question,

b) provide background and significant of the problem,

c) search for related evidence, and

d) write an evidence synthesis and clinical recommendation. Students interested in pain management in any clinical population, were invited to participate in this international education collaboration. The extra work was graded as a part of their course assignment. For this participation, students were required to

1) review information on pain management shared on Facebook closed group during October to December 2018 and discuss the information,

2) include at 1-2 clinical recommendations based on the information they learned from the Facebook,
3) shared their project finding (3-5 min presentation) on the Facebook close group, and

4) write a journal reflection of their experience at the end of the semester. Two groups ( 5 students/group) signed up for this activity.

\section{Materials and Facebook Activities}

Faculties created a Facebook closed group, which allow only members of the group to review and post comments on the discussion. Students from both schools were invited to join the group. A faculty posted a 3-minute video clip on pain management using TCM [6].

TCM Video: A 3-minute video clips regarding a client with muscular-skeletal pain on her the right hand and wrist was developed. In the video, the client stated that the pain was so severe that she said she did not want to continue her work, and, felt stressful and hopeless. Then, a health care profession introduced the use of moxibustion and applied the moxibustion on her right hand and wrist for a while. Afterwards, the client instructed to stretch her right hand and wrist again. The client stated she felt much better, and the smell of the moxibustion was indeed appealing. At the end of this video clips, the instructor as the students to discuss and share posts in the Facebook regarding the following questions.

a) To understand moxibustion, its nature, effects and side effects

b) Identify the role of moxibustion and various TCM methods in pain management

c) Explore any cultural differences in pain management between East and West populations

Facebook Discussion: Students were required to post literature-based information, sharing clinical experiences and practices on pain management in various clinical settings and populations. Faculties monitored and moderate the group discussion. From October - December 2018, there were 41 posted discussion posted by students. The topics include chronic pain in older adult and management methods in the US, the use of transcutaneous electrical nerve stimulation (TENS) for labor pain in the US, moxibustion for chronic pain, acupuncture for pediatric intensive care unit, the underlying mechanism of moxibustion and acupuncture effectiveness, the combined electroacupuncture and auricular acupuncture for post-operative pain, safety and potential complication of moxibustion, acupuncture for labor pain, cultural differences of patient's attitude toward pain, moxibustion for cancer pain and muscle pain, safety and complication of acupuncture, and Chinese complimentary therapies in labor pain management [7].

Student Reflection: At the end of the semester, students were asked to reflect on their learning experience and share comments and suggestions on what need to be done to improve the activity and the use of social media. 
Broaden knowledge: Students found that this activity broadens their nursing knowledge and experience.

a) Student A: "It was really great to see how students from Hong Kong / US actively participated. It was very structured, and they gave us a lot of education on their topics"

b) Student B: "I enjoyed reading the Hong Kong student's Facebook posts. Working as a nurse in [USA], I encounter daily patients from other cultures and countries. Reading these posts gave me a different and widened perspective of cultural sensitivity.; "Overall I think this was a meaningful experience"

c) Student C: "This course extra credit activity was very interesting and eye opening to see other students explain some of their traditional medicine techniques such as acupuncture and moxibustion."

d) Student D: "I learned that there are many other options than medications for pain relief and I learned that I need to broaden my knowledge on other cultures pain relief techniques in order to be a more well-rounded nurse."

Appreciation of Cultural Difference: Students found that the collaboration provides a new information and sharing experience and information increase their exposure to the cultural differences.

a) Student E: "Nice to hear opinions from another countries standpoint where culture is very different and things up for interpretation can be taken very differently.

b) Student F: "It was a great experience interacting with students from Hong Kong / US to better understand other means of pain management in different cultures"

c) Student G: "We have been taught over and over again about using culturally appropriate care. Working with other students and learning about their work (specifically in moxibustion that I previously knew nothing about) was eyeopening."

d) Student H: we hope other students tried acupuncture and found it effective."

Convenience of Using Facebook Platform: Some students feel that sharing the information through the Facebook close group is convenience.

a) Student C: "The most helpful aspect of this activity was the use of Facebook as the platform. Although not a traditional way to learn, it made it easy to see and read on my phone during my busy schedule of school and working full time."

b) Student D: "I think having a platform such as Facebook was helpful for this activity for us to get in contact with others. It also encouraged interaction and deliberation."

However, students suggested that there should be more structured evaluation criteria for the learning using the social media method. Some students did not use Facebook regularly and creating another social media account is a burden. Therefore, they suggested using other social media (e.g., twitter, Pinterest, etc.).

Faculties Note: The faculties felt that the social media, especially, Facebook is easy to use. This platform offers an attractive feature for connection and collaboration among students. The original plan for this collaboration was to use the "live chat" feature for students to sharing and discuss their information. However, we run into a challenge of schedule conflict and time zone differences between two countries. The discussion posts, comments and presentation were used to replace the "live chat." [8] We are testing the feasibility of this collaboration. To avoid overwhelm the students with the structured requirement, we allowed the students to volunteer to be a part of the activity. During October-December 2018, we did not encounter any technical difficulties for posting our comments and received no report of technical difficulty from students. Each student was required to share their clinical experience and post the literature-based evidence. Two faculties took moderator roles for the students' discussions. There were no evaluation criteria on how many posts required per each student. We found that some students participated in the discussion more than the others. We used the Facebook feature "at (@) user account" to ask question to specific student. This feature helped us received more responses from students who did not participate in the discussion.

\section{Discussion}

With demand for expansion of educational offerings both within and across countries has increased worldwide, and the proliferative use of social media among younger generations. The present article described the effective use of social media to enhance the learning of TCM. Indeed, management of pain is of important concern in the medical arena. All health-related professionals encounter patients with pain throughout their career. Nurses are integral to improving the health of individuals, families and communities worldwide; therefore, it is critical for nursing programs to be actively involved in addressing global health concerns by promoting a deeper understanding of cultural perspectives of different health conditions [9]. Students from both schools actively participated in the discussion and sharing relevant articles and photos and local news related to topics. Students found this activity rewarding and widening the cultural sensitivity perspective. As health becomes a global issue, the distance collaborations among health care professionals are common. Social media platforms can be used as tools to overcome scheduling issues and encourage interaction and deliberation.

In spite of the benefit on interaction and communication, the privacy and security issues of social media were major concerns [9]. We employed the security measure by setting up the privacy feature of the Facebook group to allow only group members to post and share information, the members were invited members only, and the group cannot be searched from search engine. Before started 
sharing the information, the group rules on sharing and posting information were established and agreed by group members. The project is limited by specific social media (Facebook). Not all students are regular Facebook users, and it posed a limitation of this study. For the future collaboration, we will need to explore other social media options.

In conclusion, the use of social media to embraces the technology-assisted approaches and enhance international teaching and learning experience is feasible. The teaching team will continue to develop and refine collaborative mode to facilitate students learning.

\section{Acknowledgement}

The authors thank Dr. Corjena Cheung for her contributions to the project. Sincere appreciation to the inputs from Amy Ezhaya, Michael Gerstel, Caresse Hollendoner, Rebecca Ingber, Caryn Just, Eunice Adu, Kyle Brashear, Ashby Health, Paige Martin, Melissa McCoy, and Daniel Moorhead.

\section{References}

1. Lepp M, Halabi JO, Määttä S (2011) Jordanian nursing faculty experiences of participation in international exchange programmes with Sweden. Diversity in Health and Care 8(3): 181-188.

\section{ISSN: 2574-1241}

DOI: $10.26717 /$ BJSTR.2019.19.003286

Mimi Tse. Biomed J Sci \& Tech Res

This work is licensed under Creative Commons Attribution 4.0 License

Submission Link: https://biomedres.us/submit-manuscript.php
2. Manaseer S, Alawneh A, Almogdady H (2019) Social Media as a tool in education: A content descriptive analysis. International Journal of Information, Business and Management 11 (2): 214-219.

3. Shiri A, Rathi D (2013) Twitter content categorization: A public library perspective. Journal of Information \& Knowledge Management 12 (04): 1350035.

4. Chan WS, Leung AY (2018) Use of Social Network Sites for Communication Among Health Professionals: Systematic Review. Journal of Medical Internet Research 20(3): e117.

5. Chang H, Kwon YD, Yoon SS (2011) Use of acupuncture therapy as a supplement to conventional medical treatments for acute ischaemic stroke patients in an academic medical centre in Korea. Complementary Therapies in Medicine 19(5): 256-263.

6. Mill J, Astle BJ, Ogilvie L, Gastaldo D (2010) Linking global citizenship, undergraduate nursing education, and professional nursing: Curricular innovation in the 21st century. Advances in Nursing Science 3(3): E1E11.

7. Chung YC, Lau CH, Yeoh EK, Griffiths SM (2009) Age, chronic noncommunicable disease and choice of traditional Chinese and western medicine outpatient services in a Chinese population. BMC Health Services Research 9: 207-209.

8. Lin JG, Chan YY, Chen YH (2012) Acupuncture for the treatment of opiate addiction. Evidence-based Complementary \& Alternative. Medicine article ID739045.

9. Lee D, Xu H, Lin JG, Watson K, Wu RS, et al. (2011) Needle-free electroacupuncture for postoperative pain management. EvidenceBased Complementary and Alternative. Medicine article ID 696754.

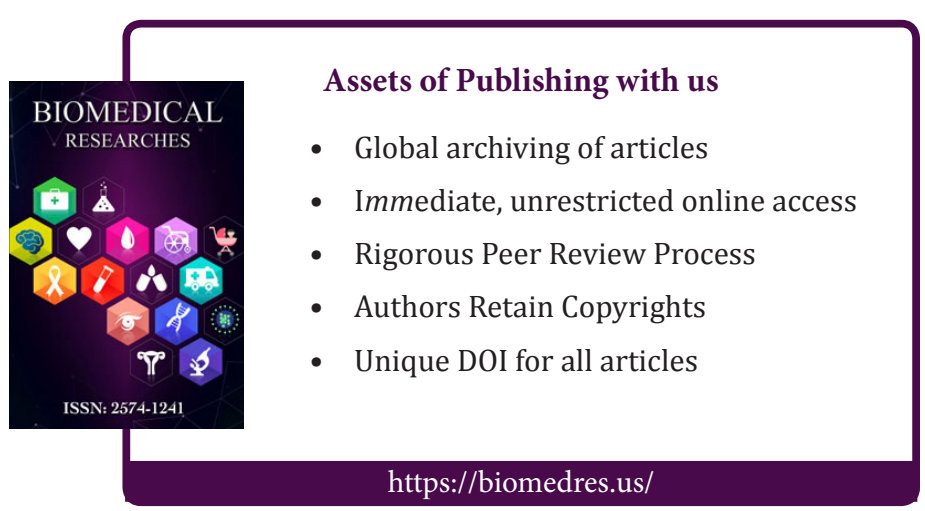

